\title{
Do background luminances interact during binocular fusion?
}

\author{
ALEXANDER I. COGAN \\ Smith-Kettlewell Eye Research Institute, San Francisco, California
}

\begin{abstract}
The question investigated in the experiments reported here was whether monocular background luminances sum during binocular fusion. Fusion was made explicit by using a random-dot stereogram (RDS) as a background stimulus. In the presence of the RDS, differential luminance thresholds were somewhat higher than in the uniform field: a full-field, binocular dot array acted as a mask for a a full-field luminance change, but global depth had no effect at threshold. The amount of the binocular advantage at threshold was compared to the basic "threshold response," that is, the change in threshold resulting from raising the background luminance by a factor of 2. It was found that the amount of the binocular advantage was equivalent, on the average, to some $75 \%$ of the threshold response-significantly less than the $100 \%$ predicted by "simple summation." The amount of the binocular advantage varied substantially among observers and eyes, whereas the threshold response obeyed Weber's law in all cases: the variability was eye-, rather than threshold-dependent. Monocular thresholds did not decrease when taken with the nontest eye occluded rather than viewing a fused background. The proposition that the adaptation state of the visual system is increased during binocular fusion (Cogan, 1982) was not supported. Yet occluding the nontest eye, rather than presenting the test stimulus monocularly against a fused background, did change monocular thresholds in some eyes and observers. These findings are interpreted as evidence for a complex binocular background interaction involving both summation and inhibition.
\end{abstract}

Monocular increment or decrement thresholds depend, of course, on the ipsilateral adapting luminance: the TvI function ${ }^{1}$ is usually a straight line in $\log$ - $\log$ coordinates. Does the contralateral background play a role? This basic question has obvious methodological significance for studies in binocular summation: while monocular thresholds are taken, should the nontest eye be deprived of all stimuli (e.g., occluded), or of only the test stimulus, with background stimuli fused? At threshold, the amount of binocular summation (also termed the binocular advantage, or superiority), as defined by the ratio $\Delta \mathrm{I}$ (monocular)/ $\Delta \mathrm{I}$ (binocular), is often near a factor of 1.4 (e.g., see Crozier \& Holway, 1939), but it is not a general constant. ${ }^{2}$ The amount of the binocular advantage may vary between the two eyes of an observer and among observers and tasks. This variability is usually attributed to differences in responses to the test pulse, but it could also arise in part from interactions related to the binocular background stimuli.

Adapting luminance is nominally the same with monocular or binocular backgrounds; implicitly, the adaptation state in the tested eye is assumed to be unaltered by the contralateral background. However, the validity

Financial support was provided by National Institutes of Health Grants RO1 EYO6578 and P30 EYO6883. My thanks are due to Hoover Chan for much help in running these long and laborious experiments, and to Maureen Clarke for programming the computer. Correspondence may be addressed to the author, Smith-Kettlewell Eye Research Institute, 2232 Webster Street, San Francisco, CA 94115. of this assumption has never been established. Cogan (1982) proposed that the adaptation state of the visual system as a whole is enhanced by background fusion. This was an ad hoc proposition, introduced as an alternative to the less plausible hypothesis of unperceived rivalry during fusion (see Levelt, 1965; Makous \& Sanders, 1978), to account for the observation that monocular sensitivity at the differential luminance threshold was lower under fusion than during the phase of rivalry dominance (Cogan, 1982; Makous \& Sanders, 1978), a finding disputed by Blake and Camisa (1978).

Much of the existing evidence suggests that monocular sensitivity at threshold is not affected by a nonrivalrous contralateral background. Crawford (1940) measured the absolute threshold while the observer's nontest eye viewed either a dark or a variously illumined uniform field; he found that contralateral luminance had no consistent effect on threshold. Cogan, Silverman, and Chan (1984) found monocular increment thresholds to be about the same when the nontest eye was occluded as when it was viewing an equiluminant background fused with that of the tested eye. Campbell and Green (1965) measured contrast thresholds as a function of spatial frequency; thresholds remained the same whether the nontest eye viewed a dark or a bright uniform field. ${ }^{3}$ In these studies, monocular stimuli at threshold appeared indifferent to the contralateral background luminance.

Nevertheless, a nonrivalrous contralateral background can affect the visual system as a whole. Lehmkuhle and Fox (1976) found that the amount of the interocular trans- 
fer of adaptation was greater if the background viewed by the nonadapted eye was illumined rather than dark. And monocular contrast thresholds are certainly affected when congruent contours are physically present (although not necessarily visible) in the contralateral background. Relative to the baseline obtained against a uniform contralateral background, contrast thresholds drop when the contralateral contrast is weak (subthreshold) and rise with dichoptic contrast, yielding the well-known "dippermasking function" (Legge, 1979). It is not clear why binocular background interactions have been found with some, but not all, of the tasks cited above.

The purpose of the present study was limited to investigating the role of stereoscopic background fusion in binocular luminance summation. In binocular summation experiments, there is usually no independent evidence of sensory fusion, since the binocular background stimuli are identical. In such conditions, fusion is only implied, by the absence of either diplopia or manifest rivalry (see Kaufman, 1974; Levelt, 1965). Are the backgrounds truly fused, or merely superimposed? Whatever the magnitude of binocular summation, one cannot very well use it as a test for sensory fusion if summation is the only indicator of the activity associated with fusion. To obtain positive evidence of sensory fusion, Cogan (1987) introduced a random-dot stereogram (RDS) as a background stimulus. (The RDS was photographed from Julesz, 1971, p. 122. A phototransparency with a random-dot array is placed in each monocular field and transilluminated. Field luminance is determined, using the $1^{\circ}$ spot of the Pritchard Spectra Spot photometer $1980 \mathrm{~A}$, as a space-average over the dot array, which has about $50 \%$ dot density.) Upon fusing the two dot arrays, the observer perceived the whole (circular) field as a three-dimensional spiral surface, receding funnel-like toward the center of the field. The contours of the spiral being Cyclopean, they exist only as long as fusion is not disrupted (see Julesz, 1971). Luminance increments or decrements, even when strongly suprathreshold, do not break global fusion (Cogan, 1989). ${ }^{4}$

The experiments reported below were conducted some years ago to determine whether explicit background fusion, a novel feature in the paradigm of binocular summation, has an effect on the amount (and the variability) of the binocular advantage at the differential threshold. These experiments elucidated the question posed in the title, but they have not answered it. The absence of a definitive answer after nearly a century of research is perhaps not surprising considering that it requires the very knowledge about the binocular function that is being sought by experiments on binocular summation.

\section{EXPERIMENT 1}

The amount of the binocular advantage at threshold was compared to the "threshold response," that is, the magnitude of threshold elevation resulting from a twofold in- crease in background luminance (see Cogan, 1983). The comparison was obtained by taking the ratio $S=$ $m(I) / b(2 I)$, where $m$ and $b$ are monocular and binocular threshold pulse amplitudes, respectively, and $I$ is binocular background luminance. The $S$ ratio was found for each eye separately. Thus, for the right-eye threshold (Condition R) and the binocular threshold (Condition B), the $S$ ratio is given by:

$$
S_{\mathrm{R}}=\Delta I_{\mathrm{R}}(I) / \Delta \boldsymbol{I}_{\mathrm{B}}(2 I) \text {. }
$$

The $S$-ratio approach is somewhat similar to the longknown "double-energy monocular" paradigm (see Blake \& Fox, 1973); both approaches test a version of the "simple-summation" hypothesis. The double-energy version assumes that energies of monocular test pulses add at threshold when stimulation is binocular (for pulse intensity, $\Delta I_{R}+\Delta I_{L}=2 \Delta I_{B}$ ); the question of binocular background interaction never arises. The $S$-ratio version of simple summation, specifically requires a binocular background and tests the assumption that all thresholds depend on its luminance, as if both the background and the test stimuli were processed in a single binocular channel. Averaged for the two eyes, the prediction of simple summation is $S=1$. In earlier studies in this laboratory (Cogan, 1983; Cogan et al., 1984), the average $S$ ratio was near unity in 6 out of 7 observers. However, with a twofold increase in adapting luminance, fortuitous similarity between the threshold response and the amount of the binocular advantage is easily possible. The binocular advantage has a range from 1 to 2 , with $\sqrt{2}$ a com mon finding. For the threshold response, one would also expect a factor of $\sqrt{2}$ with small-area stimuli, but the effect should be larger-near a factor of 2-with large-area stimuli (see Barlow, 1972). In earlier studies in this laboratory, the test field was small $\left(0.1^{\circ}\right.$ diam. $)$ and the threshold response was about a factor of 1.4 . The present experiments were conducted on a large test field, in which the threshold response could be expected to approximate a factor of 2 .

\section{Method}

Apparatus and Targets. Each eye viewed a bright circular field, $12^{\circ}$ diameter, which usually contained a random-dot array. Fused binocularly, the two arrays formed an RDS that portrayed a threedimensional spiral surface that receded toward the center of the field. Except for the disparity of the RDS, the field was identical in the two eyes. The surround was dim (about $5 \%$ of DC luminance). Luminance was produced by fluorescent lamps (Silvania F8T5/CWX) whose white light was diffused by ground-glass plates before passing through the dot arrays. (To make the field uniform, the phototransparencies that carried the RDS were simply removed.) The lamps were driven by direct current through a modified Iconix system controlled in real time by an Apple II + computer equipped with 12-bit analog-to-digital converters and a digital clock. A photocell-based feedback loop maintained DC luminance at the designated level. Test stimuli were very brief $(1-\mathrm{msec})$ rectangular pulses (rise time $0.3 \mathrm{msec}$ ) that decremented luminance uniformly over the whole field in one or in both eyes. [It has been established previously that differential thresholds for brief pulses in this field 
are identical for increments and decrements (e.g., Cogan, 1987). Decrements were used in the present experiment to ensure a sufficient range with the highest background luminance employed in the experiment.]

Procedure. Thresholds were taken with background luminance ranging from 30 to $300 \mathrm{~cd} / \mathrm{m}^{2}$. This range was subdivided into nine octaves. During one run (with any octave), monocular and binocular differential thresholds were determined in a random sequence of six conditions (right eye, left eye, and binocular; each at background luminance levels of $I$ and $2 I$ ). With each new condition, the observer adjusted the test-flash intensity to a "just visible" level, working at his or her own pace. The threshold was then determined with a two-alternative forced-choice staircase technique (after Levitt, 1970) using the just visible level as the first step of the staircase. The time necessary to complete a run was about $1 \mathrm{~h}$. ( $S$ ratios were then calculated among the six threshold values taken close together, which minimized the effect of threshold variability over time.) Nine runs formed a series covering the range of background luminance, with the sequence $\left(30 / 60,45 / 90, \ldots, 150 / 300 \mathrm{~cd} / \mathrm{m}^{2}\right)$ randomized. Six observers with normal (or corrected-to-normal) vision, 4 of them inexperienced paid volunteers, took part.

\section{Results}

In all observers, the TvI functions had a slope of unity and the binocular threshold was lower than the monocular thresholds. The TvI functions are shown in Figure 1 for 1 experienced and 1 inexperienced observer (A.C. and L.D., respectively). Figure 2 summarizes the results for

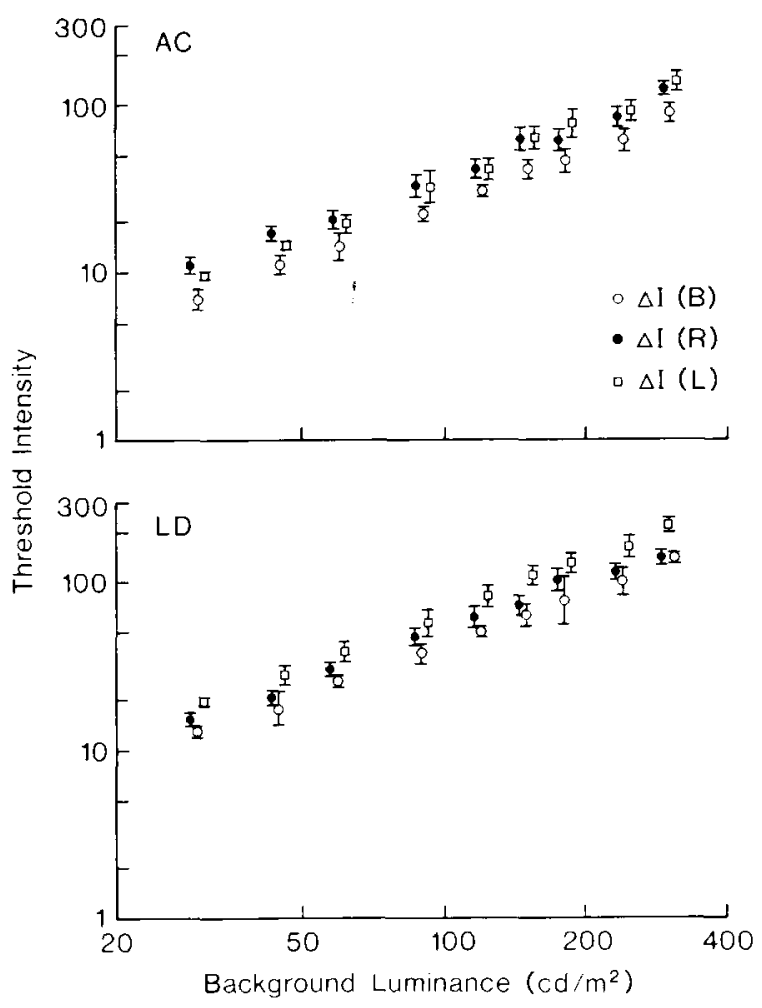

Figure 1. Monocular (R, L) and binocular (B) thresholds for Observers A.C. and L.D. as a function of background luminance with a fused, three-dimensional background field. Each point is the average of eight measurements. Error bars show $\pm 1 S D$. all observers. See also Table 1 . The threshold response was a factor of $2.06 \pm 0.10$, pooled for binocular and monocular stimuli: Weber's law very nearly held. There was significant interocular and interindividual variability in the amount of the binocular advantage-about the usual average: a factor of $1.51 \pm 0.24$. The $S$ ratio mirrored the variability of the binocular advantage ratio and was thus dependent on the eye, rather than on the threshold response. The grand mean was $S=0.745 \pm 0.11$. In 10 eyes out of $12, S$ was significantly different from unity $[F(5,11)=10.544, p<.003]$, and the average value for the $S$ ratio was always below unity, ranging from 0.64 to 0.87 among observers: see Table 1 , rightmost column.

As a control, the RDS transparencies were removed, so that the background field was uniform, and the 2 experienced observers (A.C. and H.C.) repeated the experiment. The results obtained in the absence of random-dot arrays differed from those above only in that all thresholds were lower: a factor of $1.77 \pm 0.12$ and $1.39 \pm 0.14$ for the binocular thresholds of observers A.C. and H.C., respectively. Their $S$ ratios were the same in the uniform field as in the RDS field.

\section{Discussion}

The present results, obtained with demonstrably active background fusion, did not differ from the typical findings reported in the many previous studies of binocular summation at the differential luminance threshold when background fusion was not explicitly present (reviewed by Blake \& Fox; 1973, and Blake et al., 1981). These results do not conform to either version of the hypothesis of simple binocular summation. This is not surprising, since no single-channel binocular model has been generally successful in predicting binocular threshold from monocular threshold performances (see Cogan, 1987; Cohn \& Lasley, 1976). I consider the simple-summation hypothesis a useful point of departure because it is the simplest neural hypothesis. ${ }^{5}$ On finding that summation is only partial $\left(1<\Delta I_{m} / \Delta I_{b}<2\right.$, or $0.5<S<1$ ), one is faced with numerous possible models, even at absolute threshold (see Thorn \& Boynton, 1974), where the role of binocular background interaction is not a consideration and the $S$-ratio approach does not apply. Further discussion is postponed until after some additional controls have been described, next.

\section{EXPERIMENT 2}

This experiment is complementary to Experiment 1. The issue of interaction between a monocular pulse and a binocular background was approached here from two different angles: (1) by asking whether monocular thresholds differed when measured on a fused, as opposed to a monocular, background, and (2) by inquiring into the cause of the observation made in Experiment 1, that thresholds were higher in the presence of RDS than in the uniform field. If global depth was responsible for this threshold elevation, then some form of interaction between 

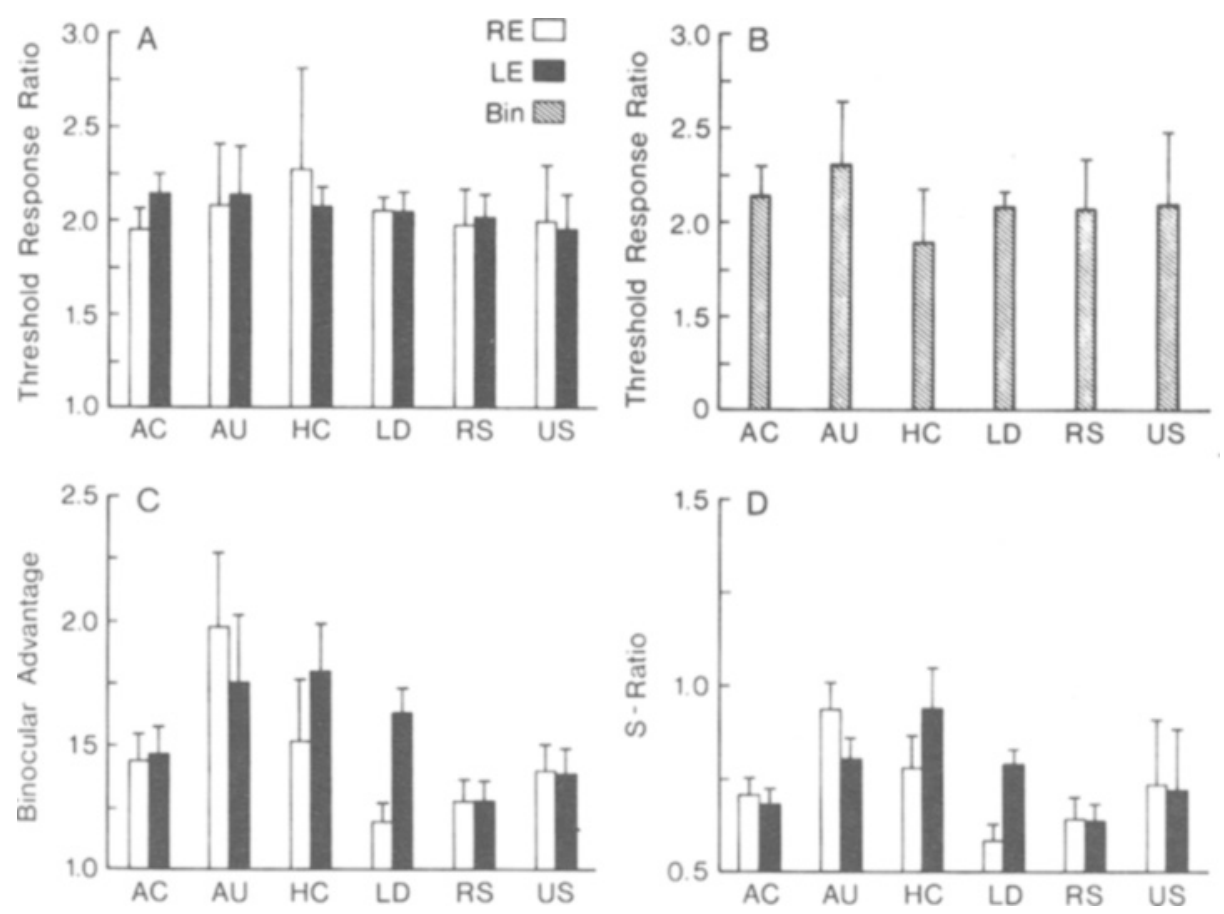

Figure 2. Mean results of monocular (A) and binocular (B) threshold responses (where the ordinate value of 2 indicates Weber's law), the mean amount of the binocular advantage at threshold (C), and the $S$ ratio (D). Error bars show 1 SD. RE and LE stand for right and left eye, respectively. All observers are identified by initials.

a fused background and a luminance change at threshold would be indicated. Alternatively, the dot arrays per se could have elevated the thresholds relative to those of the uniform field.

Thresholds were compared among the following background conditions: (1) receding spiral (RDS with uncrossed disparity); (2) protruding spiral (same RDS, but with crossed disparity); (3) a flat binocular plane (RDS with near-zero disparity (the plane looked slightly "pockmarked" due to imperfections of photoreproduction); (3M) a monocular dot array in the tested eye with the nontest eye occluded; (4) a uniform binocular field; and (4M) a uniform monocular field with the nontest eye $o c$ cluded.

\section{Method}

The procedure was the same as in Experiment 1, except that in Conditions $3 \mathrm{M}$ and $4 \mathrm{M}$ an occluder was placed in front of the nontest eye (about $12 \mathrm{~mm}$ from the cornea) whenever the computer selected a monocular condition. The occluder (a disk of mylar, $0.2 \mathrm{~mm}$ thick) was translucent, and thus the occluded eye was diffusely illuminated at about the same DC level as the tested eye. The same 6 observers were tested with the same paradigm but using only one octave of the background luminance change $\left(60 / 120 \mathrm{~cd} / \mathrm{m}^{2}\right)$.

\section{Results}

In 5 observers, thresholds were lower in the uniform field than in the presence of RDS: the threshold ratio taken between their results in Conditions 4 and 3 (binocular uni-

Table 1

Summary of Results in the Binocular Three-Dimensional Field

\begin{tabular}{|c|c|c|c|c|c|c|c|c|}
\hline \multirow[b]{2}{*}{ Observer } & \multicolumn{3}{|c|}{$\begin{array}{l}\text { Mean Response to a } \\
\text { Twofold Change in } \\
\text { Adapting Luminance } \\
\end{array}$} & \multicolumn{2}{|c|}{$\begin{array}{c}\text { Mean Binocular } \\
\text { Advantage }\end{array}$} & \multicolumn{2}{|c|}{$\begin{array}{c}S \text { Ratio } \\
(M \pm 1 S D)\end{array}$} & \multirow{2}{*}{$\begin{array}{c}\text { Mean } \\
(\mathrm{RE}+\mathrm{LE}) / 2\end{array}$} \\
\hline & B & RE & LE & $\mathrm{B} / \mathrm{RE}$ & B/LE & RE & LE & \\
\hline A.C. & 2.1 & 2.0 & 2.1 & 1.44 & 1.47 & $0.71 \pm .04$ & $0.68 \pm .02$ & 0.70 \\
\hline H.C. & 1.9 & 2.3 & 2.1 & 1.51 & 1.80 & $0.77 \pm .11$ & $0.94 \pm .11$ & 0.86 \\
\hline L.D. & 2.1 & 2.1 & 2.0 & 1.19 & 1.63 & $0.58 \pm .04$ & $0.79 \pm .04$ & 0.68 \\
\hline R.S. & 2.1 & 2.0 & 2.0 & 1.28 & 1.28 & $0.64 \pm .05$ & $0.64 \pm .04$ & 0.64 \\
\hline U.S. & 2.1 & 2.0 & 2.0 & 1.40 & 1.39 & $0.73 \pm .17$ & $0.72 \pm .16$ & 0.72 \\
\hline A.U. & 2.3 & 2.1 & 2.1 & 1.98 & 1.75 & $0.94 \pm .07$ & $0.80 \pm .05$ & 0.87 \\
\hline Mean $\pm 1 S D$ & \multicolumn{3}{|c|}{$2.06 \pm .10$} & \multicolumn{2}{|c|}{$1.51 \pm .24$} & $0.74 \pm .11$ & $0.745 \pm .10$ & \\
\hline
\end{tabular}

Note-RE $=$ right eye, $\mathrm{LE}=$ left eye, B $=$ binocular. $S$ ratio was calculated with the formula $S=(m / I) /(b / 2 I)$. Two-way analysis of variance shows that the interindividual differences among $S$-ratio scores were highly significant $(p<.001)$. 
form and binocular RDS with near-zero disparity, respectively) was equal, on the average, to $0.74 \pm 0.09$, corresponding to a $26 \%$ lower threshold in the uniform field $(t=-3.83, p<.001)$. (In Observer U.S., this comparison showed a shift in the opposite direction, with the ratio equal to $1.125 \pm 0.07, t=2.8234, p<.05$ ). The masking effect was not related to global depth. Rather, the binocular dot array per se acted as a mask for a threshold change of field luminance. It can be seen from Figure 3 that threshold differences among the RDS conditions (Conditions 1, 2, and 3) were either absent or small, relative to those found between any one of these conditions and the binocular uniform-field condition (Condition 4). In a comparison between thresholds taken in the "dotless" (Condition 4M) and "dotted" (Condition $3 \mathrm{M}$ ) monocular conditions, the masking effect was found only in Observers A.C. and L.D. (mean threshold ratio $=0.61 \pm 0.09, p<.001$ ); no effect was found in the other 4 observers (mean threshold ratio calculated among their results $=1.00 \pm 0.15$ ).

The threshold effect of occluding the nontest eye was neither large nor uniform among the eyes of different observers (Figure 3). The relevant comparisons are between monocular thresholds in Conditions 3 (binocular dots) and Condition 3M (monocular dots), and also between Condition 4 (binocular uniform) and Condition 4M (monocular uniform). With the nontest eye occluded, some observers (A.C. and A.U.) showed either no change or a slight reduction in threshold, whereas other observers showed a change, if any, in the opposite direction; thus, the right-eye threshold in Observer L.D. increased significantly when the left eye was occluded, and this effect
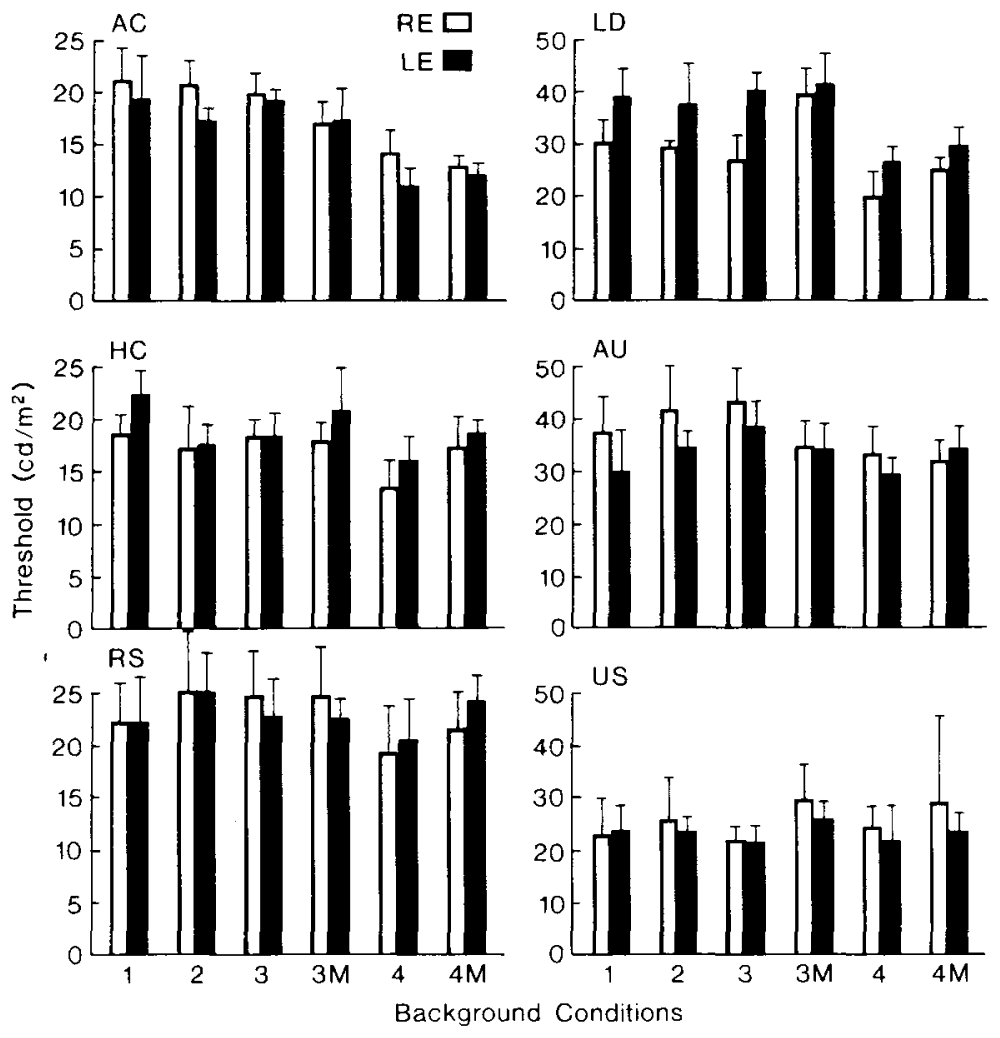

Figure 3. Right-eye (RE) and left-eye (LE) monocular thresholds. Each bar is the average of eight measurements. Eficiency difiered among observers (note the difference in scale on the ordinate). Error bars show $1 S D$. Numbers below the histograms indicate the following background conditions: (1) random-dot stereogram (RDS) with uncrossed disparity, (2) RDS with crossed disparity, (3) RDS with near-zero disparity, (3M) monocular dot array with the nontest eye occluded, (4) binocular uniform field, and (4M) monocular uniform field with the nontest eye occluded. Thresholds were not affected by the amount of disparity, nor by its sign (among Conditions 1, 2, 3), but they dropped in the absence of random-dot arrays (Condition 4), except in Observer U.S. Occlusion of the nontest eye usually caused little or no change in threshold, but sometimes resulted in a reduction (e.g., Observer A.C.) or a significant increase (e.g., Observer L.D., right eye) in thresholds: compare Observer 3M to Observer 3, and Observer $4 \mathrm{M}$ to Observer 4. 
was more pronounced in the presence of dots (compare Conditions 3 and $3 \mathrm{M}$ ) than in the uniform field (compare Conditions 4 and $4 \mathrm{M}$ ).

\section{Discussion}

The evidence concerning binocular interaction between background luminances during fusion is ambiguous. Occlusion of the nontest eye did not, as a rule, result in a reduction of monocular thresholds, and this finding goes against the proposition (Cogan, 1982) that binocular fusion enhances the adaptation state for the visual system by summing monocular background luminances. A more complex interaction than mere summation is indicated by the finding that monocular thresholds in some observers changed when the nontest eye was occluded, as has also been observed before in similar circumstances (e.g., Cogan, 1983).

These observations, taken together with other apparently discordant findings described in the introduction, suggest that a working psychophysical model of binocular background interaction must incorporate reciprocal interocular inhibition of some form. One approach might be essentially similar to the "either-eye" process (Cogan, 1987), in which the amount of binocular summation is reduced by about one half by reciprocal, interocular forward inhibition. (In its present form, Cogan's model does not incorporate background interactions; it accounts for the binocular advantage at threshold, as well as its variability, solely in terms of the magnitude of a "fused" process, a multiplicative excitatory response to a binocular test stimulus.) Applied to background luminance summation, the either-eye process would make binocular and monocular adapting luminances effectively equal, and a monocular threshold should then be the same on a fused binocular, as on a monocular, background. Furthermore, by assigning unequal weights to the eyes, it should also be possible to account for the observed differences in monocular thresholds measured on fused, as opposed to monocular, backgrounds. This outline is, of course, not yet a working model.

A possible alternative approach would be to use recurrent, rather than forward, interocular inhibition, as suggested in Lehky's (1988) model. However, it is not clear at this point whether any such model could be meaningfully related to the complex issue of light adaptation and the sensitivity of the visual system to luminance changes (see review by Hood \& Finkelstein, 1986). In any case, detailed consideration of models of binocular summation is outside of the scope of this paper.

The practical, positive outcome of these studies is that the random-dot arrays proved highly satisfactory as a background stimulus in these (and similar) experiments. In addition to serving as a fusion lock and making fusion explicit, the RDS caused considerably less discomfort when observed for long periods of time than did the same fields without the RDS, perhaps because the uniform fields left sharper and more persistent afterimages. Since there was no interference between test pulses and the background stereo stimuli, the advantages of using such stimuli at the differential luminance threshold clearly outweigh the slight loss of sensitivity that was due to the masking effect of the dot arrays.

\section{REFERENCES}

BARLow, H. (1972). Spatial and temporal summation and regulation. In D. Jameson \& L. M. Hurvich (Eds.), Handbook of sensory physiology (Vol. VII/4, pp. 9-13). New York: Springer-Verlag.

BLAKE, R., \& CAMISA, J. (1978). Is binocular vision always monocular? Science, 200, 1497-1498.

BLAKE, R., Fox, R. (1973). The psychophysical inquiry into binocular summation. Perception \& Psychophysics, 14, 161-185.

Blake, R., Sloane, M., Fox, R. (1981). Further developments in binocular summation. Perception \& Psychophysics, 30, 266-276.

Campbell, F. W., GReEN, D. G. (1965). Monocular versus binocular visual acuity. Nature, 208, 191-192.

CAvonius, C. R. (1979). Binocular interactions in flicker. Quarterly Joumal of Experimental Psychology, 31, 273-280.

Cogan, A. I. (1982). Monocular sensitivity during binocular viewing. Vision Research, 22, 1-16.

Cogan, A. I. (1983). Binocular fusion regarded as simple summation of monocular photopic luminances. Vision Research, 23, 1725-1729.

CoGAN, A. I. (1987). Human binocular interaction: Towards a neural model. Vision Research, 27, 2125-2139.

Cogan, A. I. (1989). Anatomy of a flash. 1. Two-peak masking and a temporal filling-in. Perception, 18, 243-256.

Cogan, A. I., Silverman, G., Chan H. (1984). Binocular summation of fused annular backgrounds. Perception, 13, 193-206.

Cohn, E. T., \& LASLeY, D. J. (1976). Binocular vision: Two possible central interactions between signals from two eyes. Vision Research, 192, 561-563.

Crawford, B. H. (1940). Ocular interaction in its relation to measurements of brightness threshold. Proceedings of the Royal Society of London, Series $B, 128,552-559$.

Crozier, W. J., Holway, A. H. (1939). Theory and measurements of visual mechanisms. $3, \Delta \mathrm{I}$ as a function of area, intensity, and wavelength for monocular and binocular stimulation. Joumal of General Psychology, 23, 101-141.

ENGEL, G. R. (1970). An investigation of visual responses to brief stereoscopic stimuli. Quarterly Joumal of Experimental Psychology, 22, $148-160$

Hood, D. C., Finkelstein, M. A. (1986). Sensitivity to light. In K. R. Boff, L. Kaufman, \& J. P. Thomas (Eds.), Handbook of perception and human performance: Vol. 1 . Sensory processes and perception (chap. 5, pp. 1-66). New York: Wiley.

JuLEsz, B. (1971). Foundations of Cyclopean perception. Chicago: University of Chicago Press.

Kaufman, L. (1974). Sight and mind. Oxford: Oxford University Press.

LEGGE, G. E. (1979). Spatial frequency masking in human vision: Binocular interactions. Journal of the Optical Society of America, 69, 838-847.

LEGGE, G. E. (1984). Binocular contrast summation: 1. Detection and discrimination. Vision Research, 26, 373-383.

LEHKY, S. R. (1988). An astable multivibrator model of binocular rivalry. Perception, 17, 215-228.

LeHMKUHLE, S., \& Fox, R. (1976). On measuring interocular transfer. Vision Research, 16, 428-430.

LeVelT, W. J. M. (1965). On binocular rivalry. Soesterberg, The Netherlands: Institute for Perception RVO-TNO.

LEVITT, H. (1970). Transformed up-down methods in psychoacoustics. Journal of the Acoustical Society of America, 49, 467-477.

Makous, W., \& SANDERS, R. K. (1978). Suppressive interactions between fused patterns. In J. C. Armington, J. Krauskopf, \& B. W. 
Wooten (Eds.), Visual psychophysics and physiology (pp. 167-190). New York: Academic Press.

SherRington, C. S. (1904). On binocular flicker and the correlation of activity of "corresponding" retinal points. British Journal of Psychology, 1, 26-60.

ThORN, F., BoYNTON, R. M. (1974). Human binocular summation at absolute threshold. Vision Research, 14, 445-458.

\section{NOTES}

1. Threshold plotted versus background intensity (TvI).

2. This topic has been reviewed by Blake and Fox (1973) and Blake, Sloane, and Fox (1981); but see also Legge (1984) and Cogan (1987).

3. Campbell and Green noted that this finding precluded an interpretation of the binocular advantage at threshold directly in terms of an improved signal-to-noise ratio, but their caveat has been often ignored in the later literature on binocular summation. See Blake et al. (1981) for a thorough analysis of the model.

4. Flashes used in that study were not only suprathreshold, but were also relatively long ( $500 \mathrm{msec})$, which makes it unlikely that fusion was internupted imperceptibly, since stereoscopic persistence has a time constant of only about 300 msec (Engel, 1970).

5. Historically, simple additivity was the first hypothesis of binocular interaction to be tested-by Sherrington (1904). He resorted to the opposite proposition of "monocular independence" when little difference was found between monocular and binocular performances at flicker fusion. See Cavonius (1979) for a definitive resolution.

(Manuscript received March 11, 1988; revision accepted for publication June 9, 1989.) 